\title{
Timing-jitter, optical, and mode-beating linewidths analysis on subpicosecond optical pulses generated by a quantum- dash passively mode-locked semiconductor laser
}

Ramón Maldonado-Basilio, Josué Parra-Cetina, Sylwester Latkowski, and Pascal Landais*

School of Electronic Engineering, Dublin City University, Glasnevin, Dublin 9, Ireland

*Corresponding author: landaisp@eeng.dcu.ie

Abstract Timing-jitter measurements in optically generated subpicosecond pulses by a quantum-dash passively mode-locked semiconductor laser as a function of the bias current are reported. All the measurements are retrieved from a second-harmonic-generation frequencyresolved optical gating system. A decreasing trend in the pulse width and the associated timing jitter is found with the bias current. Additionally, the optical and mode-beating linewidths are analyzed in terms of both the mode wavelength and the bias current. From our results, we can conclude that once the optical modes are phase-locked, the optical linewidth associated to every individual longitudinal mode of the device under test does not have a significant impact on the mode-beating signal on neither the pulse width nor its respective timing jitter. 
Quantum-dash mode-locked (QDash-ML) laser diodes (LDs) have attracted significant interest in optical time-domain multiplexing systems as an effective approach for all-optical short-pulse generation and all-optical clock recovery. Short-pulse generation from $40 \mathrm{GHz}$ [1] to $346 \mathrm{GHz}$ [2], as well as frequency multiplication at $270 \mathrm{GHz}$ [3] by passive mode-locking in singlesection QDash-MLLDs, have been reported. All-optical clock-recovery at $40 \mathrm{Gbps}[4,5]$ has been demonstrated by active mode locking in single-section quantum-dot epitaxies. Apart from the low power consumption and compact size, the fast carrier dynamics as well as the broad and nearly flat gain spectrum in both $1300 \mathrm{~nm}$ and $1550 \mathrm{~nm}$ are among the more attractive features of QDash/QDot ML lasers. Another important feature in such devices is the very narrow modebeating linewidth [6,7], leading to either a small timing jitter in short-pulse generation or a reduction of timing jitter up to $200 \mathrm{fs}$ in all-optical clock-recovery applications [4,5]. In spite of such remarkable achievements, to the best of the authors' knowledge, an analysis of the timing jitter associated with the optically generated pulses has not been reported under passive modelocking conditions. Indeed, the assessment of such timing jitter has significant importance when using the QDash-MLLD as a short-pulse generator at high repetition rate. The aim of this Letter is twofold: (i) to perform a detailed experimental analysis of the linewidths as-sociated with both the optical modes and the $40 \mathrm{GHz}$ mode beating and (ii) to measure the timing jitter as-sociated with the generated pulses from a single-section QDash-MLLD. After our experiment, a decreasing trend in the optical linewidths as the wavelength increases is found at a given bias. Furthermore, a decreasing trend in the timing jitter with bias current is observed, while the mode-beating signal presents a fairly constant linewidth. It can be concluded that in our laser under test all the optical modes are strongly passively mode-locked as opposed to previous analysis performed on similar laser structures, where a different strength of the passive modelocking mechanism has been observed after using a filtering scheme at the laser output [6].

Our device under test (DUT) is a 1-mm-long single-section, without phase or saturable absorber section, dc-biased, multimode QDash Fabry-Pérot (FP) semiconductor LD [1]. A brief description of the QDash-based heterostructure, as well as features and dimensions of the active core in order to achieve emission at $1.55 \mu \mathrm{m}$ have been given in [1]. From a typical collected optical power versus bias current (LI curve) characterization, it is found the laser under test presents a bias threshold of $18 \mathrm{~mA}$ and a total collected power of $4 \mathrm{~mW}$ when operating at 400 $\mathrm{mA}$ and temperature controlled at $25^{\circ} \mathrm{C}$. The collected light is recorded on a slow-response optical power meter as a function of the increasing and decreasing bias current. Based on this characterization, no hysteresis behavior is observed, confirming the standard LI dependence of a single-section FP laser.

The experimental setup utilized to characterize the linewidths and timing jitter is depicted in Fig.1. Regarding the linewidth assessment, the laser output is first coupled to an isolator (ISO) for suppressing back reflections and then to a tunable optical band-pass filter (BPF), allowing every individual optical mode (optical linewidth measurement) and pair of consecutive modes (mode-beating measurement) to be selected and analyzed. In the case of the mode-beating linewidth measurements, a $50 \mathrm{GHz}$ photodetector (PD) and an electrical spectrum analyzer (ESA) set at $1 \mathrm{MHz}$ span and $1 \mathrm{kHz}$ video bandwidth is utilized, while for the optical linewidth measurements a self-heterodyne detection (SHD) setup is implemented prior to the PD and ESA. A semiconductor optical amplifier (SOA) operating in linear regime is used to boost the optical 
modes after the BPF, al-lowing sufficient optical power to be provided to the SHD. In addition, the timing jitter associated with the optical pulses generated by our DUT is analyzed with a second-harmonic-generation (SHG) frequency-resolved optical gating (FROG) system, with temporal and wavelength resolutions of $26 \mathrm{fs}$ and $0.02 \mathrm{~nm}$, respectively. Thus the experiment is performed by measuring the linewidths (optical and mode beating), and the timing jitter of the optically generated pulses by setting the dc-bias current from $150 \mathrm{~mA}$ to $450 \mathrm{~mA}$. The timing jitter analysis is performed on the pulses generated by our DUT after a passive compression scheme, achieved with a 450-m-long single-mode fiber (SMF) [1]. Indeed, when fitted to a Gaussian shape, the pulses exhibit a minimum FWHM of $2.2 \mathrm{ps}$ and $720 \mathrm{fs}$ at the laser output and after the passive compression scheme, respectively (see inset on Fig. 2(a), the pulse profile at the MLLD output is shown in the dotted curve and after compression in the solid curve). The linewidth measurements are performed with and without the addition of such an SMF, confirming they are not affected by our compression scheme.

A typical optical spectrum collected at the DUT output is shown in Fig. 2(a). At $350 \mathrm{~mA}$, it exhibits more than 30 longitudinal modes with $0.31 \mathrm{~nm}$ inter-modal separation, giving an optical FWHM band-width of $12 \mathrm{~nm}$ centered at $1526 \mathrm{~nm}$. After tuning the BPF from the shortest 1520 $\mathrm{nm}$ to the longest wavelength $1532 \mathrm{~nm}$ falling into the $3 \mathrm{~dB}$ bandwidth of the laser spectrum, the optical linewidth as-sociated to each longitudinal mode as a function of the bias current is measured and depicted in Fig. 2(b). In this case, the BPF is set to $0.2 \mathrm{~nm}$ bandwidth, allowing the filtering of each longitudinal mode with a minimum adjacent-mode suppression of $25 \mathrm{~dB}$. Moreover, the FWHM linewidth is obtained by a Lorentzian fitting of each optical mode resolved. As it is shown in Fig. 2(b), a decreasing trend in the optical linewidth is observed as a function of the mode wave-length at a given bias current. Furthermore, a change in the magnitude of the mode linewidth is observed as a function of the bias current. For instance, it changes from $45 \mathrm{MHz}$ to $10 \mathrm{MHz}$ at a bias of $350 \mathrm{~mA}$, while a minimum mode linewidth ranging from $20 \mathrm{MHz}$ to $5 \mathrm{MHz}$ is achieved at $250 \mathrm{~mA}$ (limited to a resolution of $17 \mathrm{kHz}$ set by the used SHD setup).

In the rf domain, a Lorentzian line shape corresponding to the mode-beating signal is measured from our DUT as a photocurrent on the fast PD and depicted in Fig. 3(a) for a bias current of 350 $\mathrm{mA}$. After tuning the BPF within the same range as that of the optical mode linewidth measurement, a fairly constant profile of the mode-beating FWHM line-width is observed. Indeed, it fluctuates from $10 \mathrm{kHz}$ to $25 \mathrm{kHz}$ regardless of the bias supplied, as shown in Fig. 3(b). To retrieve these data, the filter is tuned so that a pair of adjacent optical modes passes through it, allowing a proper detection of the mode-beating signal on the PD. In addition, the mode-beating linewidth is obtained by a Lorentzian fitting of the photocurrent measurements. A maximum line-width of $25 \mathrm{kHz}$ is measured when the entire optical spectrum (without the BPF) is considered. The behavior of the sharp mode-beating linewidth with re-spect to the mode wavelength implies that all the longitudinal modes are strongly mode-locked, regardless of the mode position on the optical spectrum and the dc-bias current supplied to the device.

Finally, the width of the optical pulses generated by the passively MLLD under test, as well as the as-sociated timing jitter (both of them retrieved from the FROG system), are shown in Fig. 4 
as a function of the bias current. In the case of the timing jitter $\tau_{\mathrm{j}}$, it is calculated by considering the intensity auto- and cross-correlation traces according to the relation

$\tau_{J}=\frac{\tau_{P}}{\tau_{a c}} \sqrt{{\tau_{c c}{ }^{2}-\tau_{a c}^{2}}^{2}}$ where $\tau_{a c}$ and $\tau_{c c}$ are the FWHM of the auto- and cross-correlation pulses, respectively.

The time-domain approach implemented for measuring the timing jitter is quantitatively comparable to the frequency-domain technique. Its advantage is that it requires neither an extremely fast photodetector nor a so-called timing jitter analyzer. As can be observed in Fig. 4, the pulse width tp and associated tj present a decreasing trend with the dc bias. For instance, $\mathrm{tj}$ associated with the widest measured pulse (tp $=1.6 \mathrm{ps}$, obtained at $150 \mathrm{~mA}$ ) is above $350 \mathrm{fs}$, while $\tau_{J}$ associated to the shortest pulse ( $\mathrm{tp}=720 \mathrm{fs}$, obtained in the range from 300 to $450 \mathrm{~mA}$ ) is $150 \mathrm{fs}$. This might imply that optical modes have altogether a better quality of their respective phase-locking mechanism at dc-bias conditions ranging from 300 to $450 \mathrm{~mA}$ than the phase locking obtained at a lower bias current.

Notice that even though the $25 \mathrm{kHz}$ mode-beating linewidth of the DUT is inferior with respect to the narrowest linewidth obtained from a 4-mm-long two-section passively mode-locked QD de-vice at $10 \mathrm{GHz}$ [7], its performance is comparable in terms of the timing jitter (minimum of $150 \mathrm{fs}$ with respect to $147 \mathrm{fs}$ reported in [7]). Moreover, considering a collected power of $4 \mathrm{~mW}$ at our DUT output with $\tau_{p}=2.2 \mathrm{ps}$ at $40 \mathrm{GHz}$, a peak power of $40 \mathrm{~mW}$ is estimated, while for compressed pulses of $720 \mathrm{fs}$ the peak power is $140 \mathrm{~mW}$. Such measurements are comparable with previous work, where a peak power of $2.25 \mathrm{~W}$ has been estimated from a 2.4-mm-long twosection mode-locking tapered QD device whose out-put power is $15.6 \mathrm{~mW}$ and $360 \mathrm{fs}$ pulses are generated at $17 \mathrm{GHz}[8]$.

In summary, a detailed experimental analysis of the linewidths and the timing jitter associated with the short pulses generated from a QDash-MLLD has been achieved. Once the optical modes altogether are mode-locked, the optical linewidth associated to every individual longitudinal mode of the DUT does not have a significant impact on the mode-beating signal neither on the pulse width nor on its respective timing jitter. In addition, the performance of the analyzed device as an all-optical short pulse generator is comparable with some other MLLDs reported in the literature in terms of pulse width, timing jitter, and output power.

The authors acknowledge Dr. Douglas Reid for fruitful discussions on the timing jitter measurements, Science Foundation Ireland, and Enterprise Ireland for their support under the projects 05/RPF/ ENG0040 and TD/2009/0303, respectively. 


\section{References}

1.S. Latkowski, R. Maldonado-Basilio, and P. Landais, Opt. Express 17, 19166, (2009).

2. K. Merghem, A. Akrout, A. Martinez, G. Aubin, A. Ramdame, F. Lelarge, and G.-H. Duan, Appl. Phys. Lett. 94, 021107 (2009).

3. M. Xia, C. H. Kwok, M. G. Thompson, R. V. Penty, I. H. White, F. van Dijk, A. Enard, F. Lelarge, and G.-H. Duan, in Conference on Lasers and Electro-Optics/ International Quantum Electronics Conference, OSA Technical Digest (CD) (Optical Society of America, 2009), paper JThE15.

4. J. Renaudier, B. Lavigne, F. Lelarge, M. Jourdran, B. Dagens, O. Legouezigou, P. Gallion, and G.-H. Duan, IEEE Photon. Technol. Lett. 18, 1249 (2006).

5. X. Tang, J. C. Cartledge, A. Shen, A. Akrout, and G.-H. Duan, Opt. Lett. 34, 899 (2009).

6. A. Shen, J.-G. Provost, A. Akrout, B. Rousseau, F. Le-large, O. Legouezigou, F. Pommereau, F. Poingt, L. Legouezigou, G.-H. Duan, and A. Ramdane in Optical Fiber Communication Conference and Exposition and the National Fiber Optic Engineers Conference, OSA Technical Digest (CD) (Optical Society of America, 2008), paper OThK1.

7. G. Carpintero, M. G. Thompson, R. V. Penty, and I. H. White, in Conference on Lasers and Electro-Optics/ International Quantum Electronics Conference, OSA Technical Digest (CD) (Optical Society of America, 2009), paper CMK2.

8. M. G. Thompson, R. V. Penty, and I. H. White, in 21st International Semiconductor Laser Conference (2008), paper MC3, pp. 27-28. 
Figures

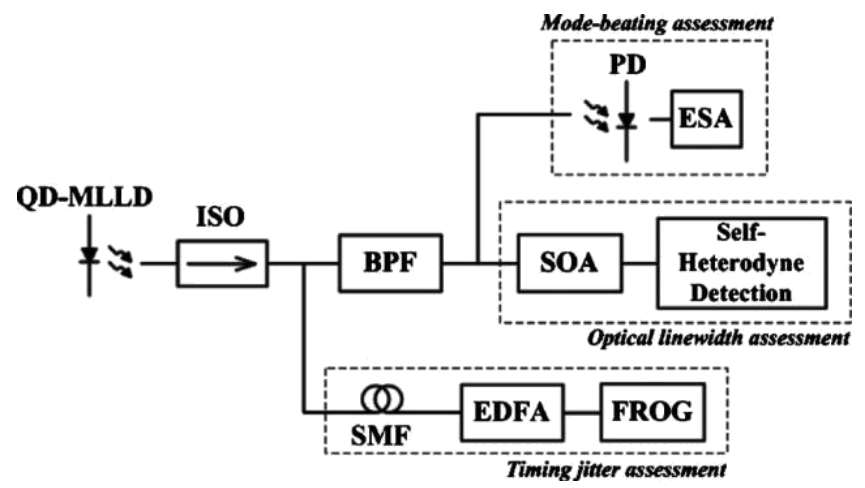

Figure 1Schematic of the experimental setup. Abbreviations are defined in the text. 

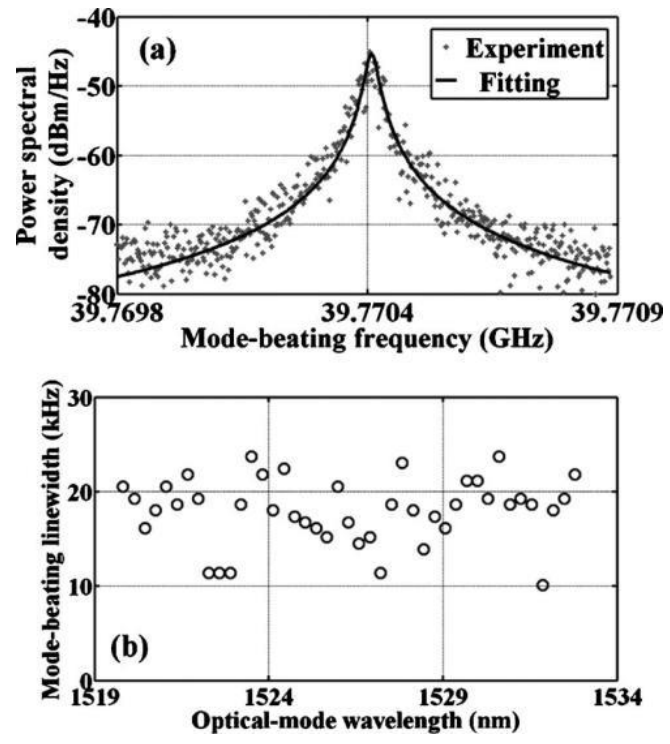

Figure 2(a) Typical optical spectrum collected from the QDash-MLLD at $350 \mathrm{~mA}$ and (b) mode linewidth measurements as a function of the bias current. In the inset, pulse profile at the output of the QDash-MLLD (dotted curve) and after passive compression (solid curve). 

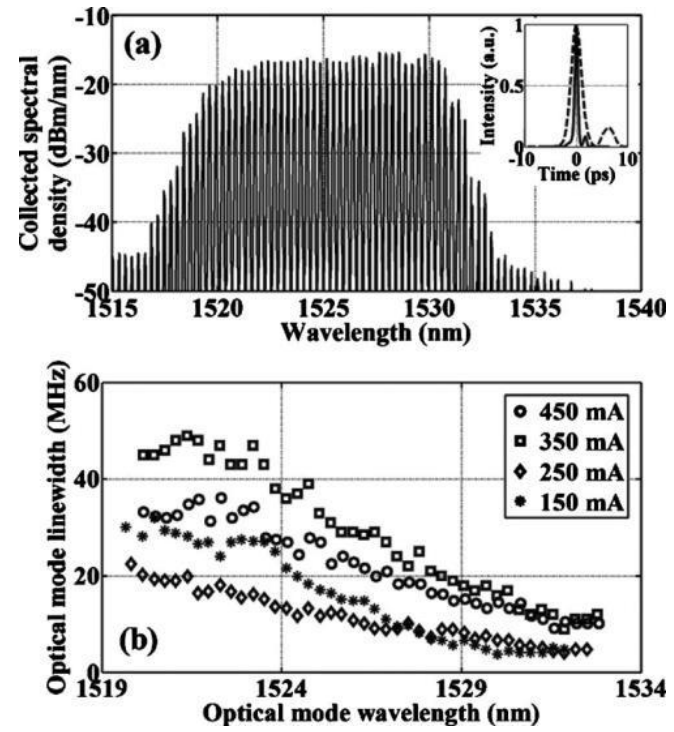

Figure 3 (a)Typical mode-beating signal measured at the output of our QDash-MLLD at $350 \mathrm{~mA}$ and (b) mode-beating linewidth associated with each pair of optical modes as a function of the BPF central wavelength. 


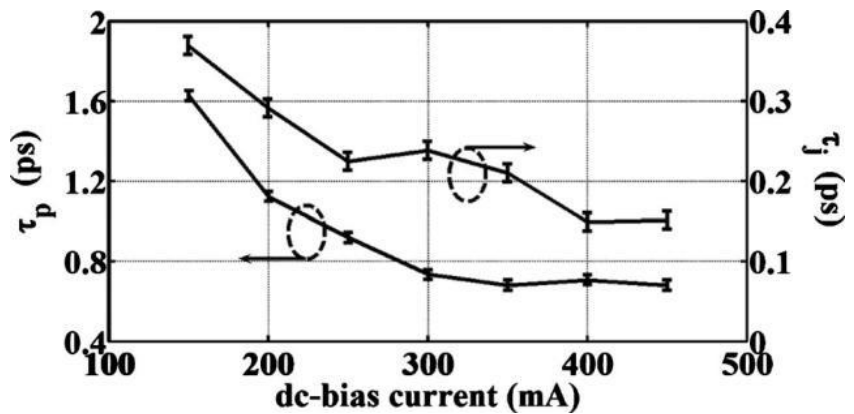

Figure 4 Pulse width tp and timing jitter tj associated with the optically generated pulses in terms of the dc-bias current supplied to the DUT. 